\title{
EGOFET Peptide Aptasensor for Label-Free Detection of Inflammatory Cytokines in Complex Fluids
}

Marcello Berto, Chiara Diacci, Roberta DAgata, Marcello Pinti, Elena Bianchini, Michele Di Lauro, Stefano Casalini, Andrea Cossarizza, Magnus Berggren, Daniel Simon, Giuseppe Spoto, Fabio Biscarini and Carlo A. Bortolotti

The self-archived postprint version of this journal article is available at Linköping University Institutional Repository (DiVA):

http://urn.kb.se/resolve?urn=urn:nbn:se:liu:diva-160145

N.B.: When citing this work, cite the original publication.

This is a copy of the published version of the following article:

Berto, M., Diacci, C., DAgata, R., Pinti, M., Bianchini, E., Di Lauro, M., Casalini, S., Cossarizza, A., Berggren, M., Simon, D., Spoto, G., Biscarini, F., Bortolotti, C. A., (2018), EGOFET Peptide Aptasensor for Label-Free Detection of Inflammatory Cytokines in Complex Fluids, ADVANCED BIOSYSTEMS, 2(2), 1700072. https://doi.org/10.1002/adbi.201700072

which has been published in final form at:

Original publication available at:

https://doi.org/10.1002/adbi.201700072

Copyright: Wiley-Blackwell

http://wiley.com/ 


\title{
WILEY-VCH
}

DOI: $10.1002 /(($ please add manuscript number))

\section{Article type: full paper}

EGOFET peptide aptasensor for label-free detection of inflammatory cytokines in complex fluids

Marcello Berto, Chiara Diacci, Roberta D'Agata, Marcello Pinti, Elena Bianchini, Michele Di Lauro, Stefano Casalini, Andrea Cossarizza, Magnus Berggren, Daniel Simon, Giuseppe Spoto, Fabio Biscarini*, Carlo A. Bortolotti*

Dr. Marcello Berto, Chiara Diacci, Prof. Marcello Pinti, Dr. Elena Bianchini, Dr. Michele Di Lauro, Dr. Stefano Casalini, Prof. Fabio Biscarini, Dr. Carlo A. Bortolotti

Dipartimento di Scienze della Vita, Università di Modena e Reggio Emilia, Via Campi 103, 41125 Modena, Italy.

E-mail: fabio.biscarini@unimore.it, carloaugusto.bortolotti@unimore.it

Dr. Marcello Berto present address: Dipartimento di Scienze Biomediche e Chirurgico Specialistiche, Università di Ferrara, Via Fossato di Mortara 17, 44121 Ferrara, Italy Dr. Stefano Casalini present address: Institut de Cieńcia de Materials de Barcelona (ICMABCSIC), 08193 Bellaterra, Spain

\section{Prof. Andrea Cossarizza}

Dipartimento di Scienze Mediche e Chirurgiche Materno-Infantili e dell'Adulto, Università di Modena e Reggio Emilia, Via Campi 287, 41125 Modena, Italy

Prof. Magnus Berggren, Dr. Daniel Simon Laboratory of Organic Electronics, Department of Science and Technology, ITN, Linköping University, S-601 74 Norrköping, Sweden

Dr. Roberta D'Agata and Prof. Giuseppe Spoto

Dipartimento di Scienze Chimiche, Università di Catania, V.le A. Doria 6, Catania

Prof. Giuseppe Spoto

Consorzio Interuniversitario di Ricerca in Chimica dei Metalli nei Sistemi Biologici, c/o Dipartimento di Scienze Chimiche, Università di Catania, Viale Andrea Doria 6, Catania, Italy

Keywords: organic bioelectronics; peptide aptamers; Affimer protein; TNF $\alpha$; binding constant

\begin{abstract}
Organic electronic transistors are rapidly emerging as ultra-high sensitive label-free biosensors suited for point of care or in-field deployed applications. Most organic biosensors reported to date are based on immunorecognition between the relevant biomarkers and the immobilized antibodies, whose use is hindered by large dimensions, poor control of sequence and relative instability. Here, we report an
\end{abstract}




\section{WILEY-VCH}

Electrolyte Gated Organic Field Effect Transistor (EGOFET) biosensor where the recognition units are surface immobilized peptide aptamers (Affimer ${ }^{\mathrm{TM}}$ proteins) instead of antibodies. We demonstrate our peptide aptasensor for the detection of the pro-inflammatory cytokine Tumor Necrosis Factor alpha $(\mathrm{TNF} \alpha)$ with a $1 \mathrm{pM}$ limit of detection. Ultra-low sensitivity is met even in complex solutions such as cell culture media containing $10 \%$ serum, demonstrating the remarkable ligand specificity of our device. The device performances, together with the simple one-step immobilization strategy of the recognition moieties and the low operational voltages, all prompt EGOFET peptide aptasensors as candidates for early diagnostics and monitoring at the point-of-care.

\section{Introduction}

Assays based on the response of an optically active label have long been the golden standard of biosensing, because of their ultra-high sensitivity and flexibility in hybridizing with probes for specific recognition. An optical sensing platform, though, bears an inherent lack of portability, the need of processing steps including separation and amplification, and the fact that it relies on fluorescent or radioactive expensive labels, which may also significantly alter the device response. Label-free electronic biosensors overcomes these shortcomings, ${ }^{[1]}$ however they usually do not match the performances of Enzyme-Linked ImmunoSorbent Assay (ELISA) or Surface Plasmon Resonance (SPR), despite the inherent amplification endowed by the field effect. In organic electronic biosensors (based on the organic field effect transistors, OFETs, architecture) the probes are assembled at one of the device interfaces, either the channel ${ }^{[2-7]}$ or one of the electrodes. ${ }^{[8,9]}$ These devices can be operated either in accumulation mode as Electrolyte Gated Organic Field Effect Transistors (EGOFETs) or in depletion mode as organic electrochemical transistors (OECTs). They combine several attractive features as ultra-high sensitivity upon direct target-probe hybridization: manufacturing with low-cost, large-area, low-temperature processes; integration into in field deployed or POC platforms that do not require specialists. In recent years, significant advances have pushed OFETs to the forefront of (bio)sensing: ${ }^{[8,10-16]}$ their sensitivity is now 


\section{WILEY-VCH}

comparable to that exhibited by state-of-the-art approaches, ${ }^{[5,12,17]}$ yet still ensuring all the other inherent advantages in terms of label-free detection, low-power consumption and operability in aqueous environment.

Among these, EGOFETs are very promising candidates for point-of-care monitoring. ${ }^{[6,8,9,18-23]}$ In EGOFETs, an electrolyte solution between an organic semiconductor thin film and the gate electrode acts as dielectric layer (Figure 1). The charge density in the semiconductor channel is modulated by the gate potential, and due to the tens of $\mu \mathrm{F} \cdot \mathrm{cm}^{-2}$ dielectric capacitance of the electrical double layers, operations can be driven at voltages smaller than 0.5 V. ${ }^{[24]}$

We recently demonstrated EGOFET immunosensors for the detection of pro- and antiinflammatory cytokines. ${ }^{[18,23]}$ The sensing unit was an Au gate electrode functionalized via a two-step strategy consisting of i) adsorption of a layer of recombinant His-Tagged Protein G, followed by ii) immobilization of the recognition antibody (Ab), whose Fc fragment binds Protein G. In this manner, a substantial uniform coverage (estimated around 30\%) and a controlled orientation of surface-bound viable antibodies are both achieved. This strategy has proven effective in detecting cytokines even in complex media. ${ }^{[23]}$ However, the antibody's large size is a limit to obtain a high surface density of recognition sites. The biorecognition layer is as thick as a few tens of nanometers, which was considered a theoretically insurmountable obstacle to detection, as biorecognition takes place way beyond the Debye screening length. ${ }^{[2,25,26]}$ The possibility to detect antigens even at distances larger than 15-20 $\mathrm{nm}$ from the transistor interface opens questions about how the mechanism of coupling between the biorecognition site and the active layer actually works. A change of capacitance at the electrolyte/biosensing interface upon hybridization has been invoked to explain the observations beyond the Debye length. ${ }^{[6,22,23]}$ Other drawbacks of the use of immunoglobulinbased scaffolds as core elements of the sensing unit are: their high molecular weight, the poor control of their sequence, their time instability and, in view of biosensors to be implanted in vivo, potential immunogenicity, besides requiring the use of animal models for their 


\section{WILEY-VCH}

development. ${ }^{[27-32]}$ This has led to an increasing interest in the search of smaller binding proteins that might replace antibodies. Among these, peptide aptamers (or Affimers ${ }^{\mathrm{TM}}$ ) are emerging as one of the potential alternative to antibodies. ${ }^{[33-35]}$ Their implementation as the core recognition element of the sensing unit of a device yields highly sensitive and remarkably stable peptide or protein aptamer-based biosensors (peptide aptasensors hereafter). To date, they have been used mostly in impedance measurements, ${ }^{[36-39]}$ or as dielectric or semiconductive layer in solid-state FETs, ${ }^{[40-45]}$ while only a few examples of organic electronics aptasensors were reported, featuring DNA aptamer immobilized on gold nanoparticles, ${ }^{[2,12]}$ or on metal electrodes. ${ }^{[46]}$

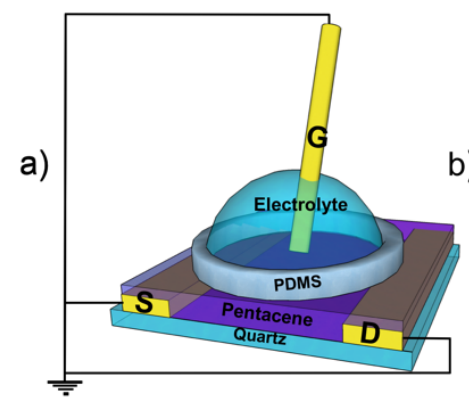

b)

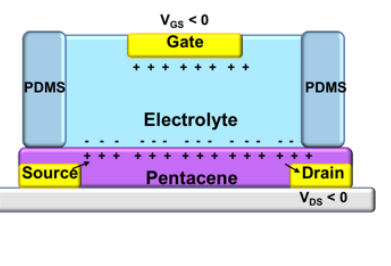

c)
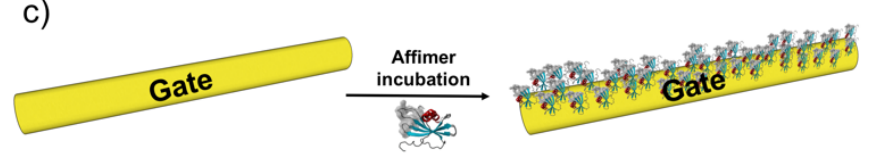

Figure 1. (a) schematic drawing of the experimental setup with electrical connections and (b) schematic of the EGOFET cross section. (c) Functionalization of the gate electrode: antiTNF $\alpha$ Affimers are directly immobilized on the gold surface by means of the His-tag.

Here, we report an EGOFET peptide aptasensor for the ultra-sensitive detection of the proinflammatory cytokine Tumor Necrosis Factor alpha (TNF $\alpha)$ down to a $1 \mathrm{pM}$ LOD value not only in aqueous buffered solution but also in cell culture medium. To the best of our knowledge, this is the first example of an organic electronic biosensor exploiting peptide aptamers as the core sensing unit on the gate electrode. TNF $\alpha$ is a cytokine plays a crucial role in mediating the inflammatory response. ${ }^{[47]}$ Although it is implicated in physiological 


\section{WILEY-VCH}

processes (apoptosis, cell proliferation and morphogenesis), altered levels are linked to a large number of diseases, including cancer, autoimmune, cardiovascular, neurologic, pulmonary, and metabolic disorders. ${ }^{[47]}$ The quantification of TNF $\alpha$ and, more broadly, of cytokines provides essential information regarding the diagnosis, stage, and prognosis of such diseases, and is routinely used in mechanistic investigation of pathological pathways. Although commercial sensing platforms for measuring TNF $\alpha$ levels are available, an alternative, labelfree and cost-effective method to quantify this cytokine in bodily fluids therefore represents a significant step towards the high-throughput, frequent screening of patients, a crucial requirement for early diagnose and personalized medicine.

\section{Results and discussion}

The schematics of our TNF $\alpha$ peptide aptasensor are depicted in Figure 1. The Au top gate electrode is electrically coupled to a $15 \mathrm{~nm}$-thick (10 monolayers) pentacene semiconductor film via a phosphate buffer solution (PBS) confined by a polydimethylsiloxane PDMS pool (see Figure S1). The gate was functionalized in a single-step procedure by incubating it with a $0.25 \mathrm{mg} / \mathrm{ml}$ solution of the peptide aptamer for $12 \mathrm{~h}$ at room temperature. The binding of the peptide aptamers to the gate was achieved by the interaction between the His-Tag at the protein C-terminus and the gold surface ${ }^{[48,49]}$ (Figure 1c). After thorough rinsing, the gate was incubated in a $100 \mu \mathrm{g} / \mathrm{ml}$ BSA (Bovine Serum Albumin) solution for $1 \mathrm{~h}$ at room temperature, in order to passivate bare gold spots that may remain after Affimer ${ }^{\mathrm{TM}}$ functionalization. $^{[50,51]}$

Electrochemical investigations were performed to quantify the gate electrode coverage with protein aptamer, resulting in a reproducible (within 10\%) surface coverage of about 2 pmol $\mathrm{cm}^{-2}\left(\right.$ viz. $\left.\sim 35 \mathrm{ng} \mathrm{cm}^{-2}\right)$.

The morphologies of bare gold electrode and gold functionalized with Affimer ${ }^{\mathrm{TM}}$ were analyzed by semi-contact mode AFM in air, and the resulting topographies are shown in 


\section{WILEY-VCH}

Figure S2 (Supporting Information). The average surface roughness and the average heights of the analyzed samples are given in Table 1: immobilization of the peptide aptamer on the gate causes an increase of the surface roughness, and a difference of the average height of

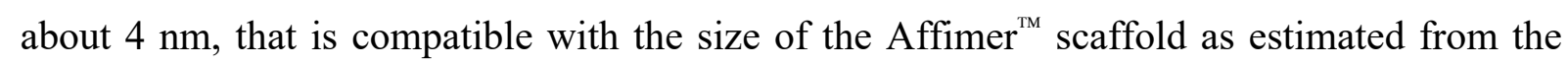
three-dimensional structure of human cystatin C (3GAX PDB). ${ }^{[52]}$

Table 1: Surface roughness and average heights of bare gold electrode and functionalized electrode.

\begin{tabular}{|c|c|c|}
\hline & Roughness (nm) & Height $(\mathrm{nm})$ \\
\hline Bare gold & $0.75 \pm 0.06$ & $7.1 \pm 0.6$ \\
\hline Affimer $^{\mathrm{TM}}$ & $3.3 \pm 1.6$ & $11.1 \pm 3.0$ \\
\hline
\end{tabular}

The device response towards TNF $\alpha$ was monitored by recording the transfer characteristics ( $I_{D S}$ vs $V_{G S}$ at a fixed $V_{D S}=-0.1 \mathrm{~V}$ ) first in PBS only and then upon incubation of the gate electrode in PBS solutions containing increasing $\mathrm{TNF} \alpha$ concentrations, spanning four orders of magnitude from $1 \mathrm{pM}$ to $10 \mathrm{nM}$ (see Figure 2a). The current $\mathrm{I}_{\mathrm{DS}}$ decreases as the [TNF $\alpha$ ] increases, and we also observe a decrease in the slope of the linear region of the transfer characteristics. In order to construct the corresponding EGOFET peptide aptasensor calibration curve, we define the response signal $S=-\Delta I / I_{0}$, i.e. the change in the device current normalized by the current in the absence of analyte $\left(I_{0}\right)$, at a given $V_{G S}$. S was then plotted as a function of [TNF $\alpha$; in Figure 2b, the dose curve at $V_{G S}=-0.5 \mathrm{~V}$ is shown.

EGOFETs are multi-parametric devices: the effect of the biorecognition event at the gate can also impact on the transconductance $g_{m}$, i.e. the product of charge carriers mobility $\mu$ times the effective capacitance $C_{\text {eff }}\left(g_{m}=\mu C_{e f f}\right)$, and the threshold voltage $V_{t h .}{ }^{[14,53]}$ We chose to monitor the analyte levels through the relative variation in $I_{D S}$, since, in view of high-throughput or in field-deployed applications, it is technologically more straightforward to develop a device 


\section{WILEY-VCH}

providing a current value as output, rather than requiring the acquisition of a full transfer curve and numerical fitting to extract parameters such as $g_{m}$ or $V_{t h}$.

The changes in $g_{m}$ and $V_{t h}$ as a function of $[\mathrm{TNF} \alpha]$ are shown in Figure 2c and $2 \mathrm{~d}$, respectively. The $g_{m}$ trend closely mirrors that of the current variation $S$, while the trend between $V_{t h}$ and the analyte concentration is more complex. ${ }^{[54]}$

a)

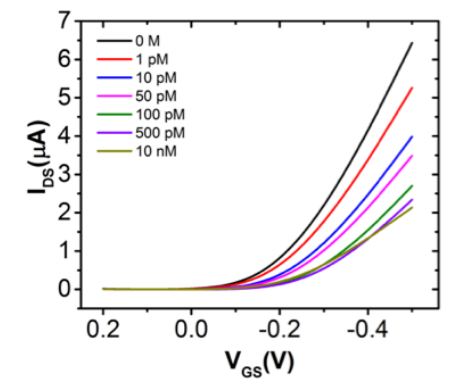

b)

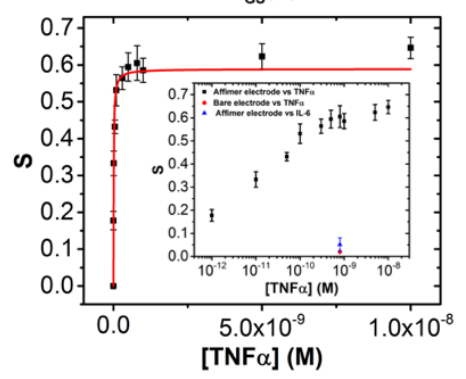

c)

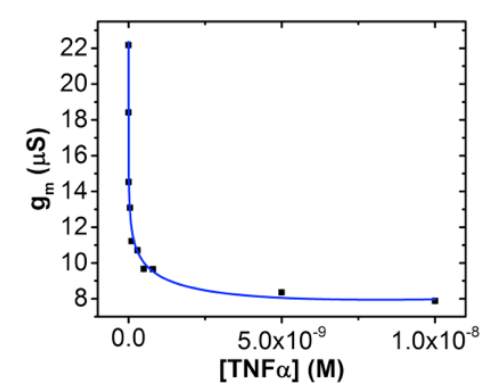

d)

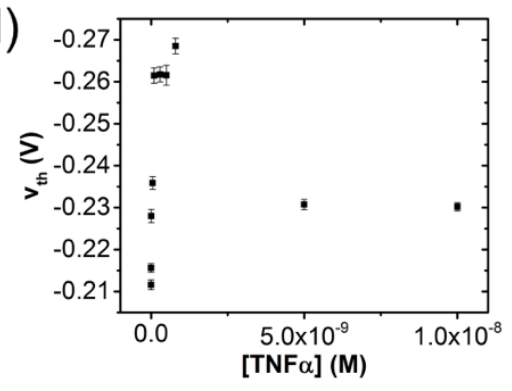

Figure 2. a) Transfer characteristics of the EGOFET-based sensor resulting from exposure to different $[\mathrm{TNF} \alpha]$ solutions in PBS (TNF $\alpha$ concentrations are reported in the legend). (b) Dose curve $S$ vs $[\mathrm{TNF} \alpha]$ acquired at $V_{G S}=-0.5 \mathrm{~V}$. The continuous red line is the best fit with Langmuir functional; inset shows the first selectivity tests: a comparison between the specific response of the aptasensor towards TNF $\alpha$ ranging from $1 \mathrm{pM}$ to $10 \mathrm{nM}$ (black) and the 


\section{WILEY-VCH}

negative control experiment towards Interleukin-6 (IL-6) 800 pM (blue), and the response of a non-functionalized gate electrode (i.e. bare gold) towards $800 \mathrm{pM} \mathrm{TNF \alpha} \mathrm{(red).} \mathrm{(c)}$ Transconductance of EGOFET-based sensor as a function of [TNF $\alpha$ ]; solid blue line is the best fit using Langmuir functional. (d) Variation of threshold voltage vs [TNFa] for EGOFET-based sensor. The error bars correspond to the r.m.s. of $S$ averaged over eight devices measured from two different test patterns.

Up to [TNF $\alpha] 1 \mathrm{nM}$, both $g_{m}$ and $V_{t h}$ trends show the same behaviour of signal $\mathrm{S}$, as reported in ref. [23]; nonetheless, at higher $\mathrm{TNF} \alpha$ concentration, $V_{\text {th }}$ decays via a slower power law towards a less negative plateau. It is difficult to disentangle the contributions of $V_{t h}$ and $g_{m}$ to this complex behaviour: our hypothesis is that at lower concentrations the binding of TNF $\alpha$ to the immobilized Affimer ${ }^{\mathrm{TM}}$ molecules leads to a decrease of the interfacial capacitance between gate and electrolyte, directly affecting $C_{e f f}^{[55]}$ and, subsequently, $g_{m}$. This would cause an increase of the voltage drop across this interface, resulting in the lowering of the modulus of the effective gating potential, and eventually in a negative shift of $V_{t h}$. Moving to the $\mathrm{nM}$ concentration range, subsequent analyte additions do not impact on $C_{\text {eff }}$, as it is shown by the quasi-plateau reached by both $S$ and $g_{m}$; this might indicate a complete coverage of the available sites on the gate surface by a layer of TNF $\alpha$. The observed $V_{t h}$ trend above $1 \mathrm{nM}$ can be rationalized in terms of charge distribution: at the operational $\mathrm{pH}$ the charge of TNF $\alpha$ is slightly negative, being its isoelectric point 6.44 , and these additional charges could constitute an additive contribution to the effective gating potential, positively shifting $V_{t h}$.

Analysis of the dose curve in Figure 2a shows that the signal $S$ rises very steeply at [TNF $\alpha$ ] $<100 \mathrm{pM}$, then sets into a linear or slowly varying region. Based on the standard deviation of the response of blank samples (i.e. PBS only or cell culture medium only), we determined the limit of detection (LOD) to be as low as $1 \mathrm{pM}$. Plasma TNF $\alpha$ levels under physiological conditions may vary among individuals, but usually lie in the $1-10 \mathrm{pg} / \mathrm{mL}\left(1-10 \cdot 10^{-14} \mathrm{M}\right)$ range and raise significantly to the picomolar range under pathological conditions related to a 


\section{WILEY-VCH}

large number of diseases, such as leukemia, rheumatoid arthritis, HIV infection, obesity, psoriasis. ${ }^{[56-61]}$

The presence of two distinct response regions suggests that the device sensitivity (which corresponds to the slope of the sensor response) is not constant throughout the investigated range.

We used surface plasmon resonance imaging (SPRI) to achieve an additional evaluation of the interaction between $\mathrm{TNF} \alpha$ and Affimer ${ }^{\mathrm{TM}}$. With this aim, we reproduced the experimental conditions used to prepare the reactive surface of the EGOFET peptide aptasensors. Figure 3 shows the SPRI signal change over time detected during the incubation of the SPRI sensor gold surface in anti-TNF $\alpha$ Affimer ${ }^{\mathrm{TM}}$.

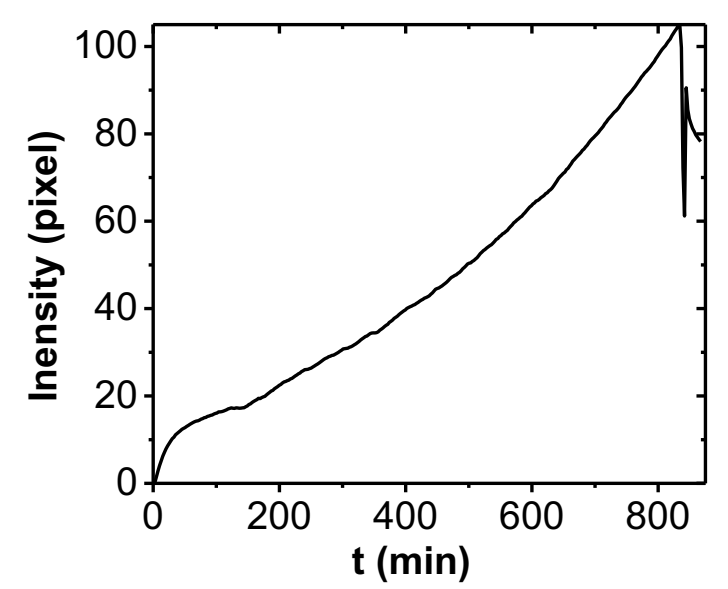

Figure 3: SPRI signal change over time recorder during the immobilization of Affimer ${ }^{\mathrm{TM}}$ on gold surface. The immobilization was obtained by incubating the gold surface in anti-TNF $\alpha$ Affimer $^{\mathrm{TM}}(0.25 \mathrm{mg} / \mathrm{ml}$ in PBS $-100 \mathrm{mM}, 75 \mathrm{mM} \mathrm{NaCl}, 0.02 \%$ sodium azide- at $\mathrm{pH} 6.5)$ for 12 h. A change of the detected signal of about 90 pixels (0-255 scale) was achieved after the immobilization. After about 130 minutes a change in the time dependence of the adsorption process is observed.

The pixel signal change (about 80 on a 0-255 scale) confirmed that the adopted protocol provided an effective immobilization of the anti-TNF $\alpha$ Affimer ${ }^{\mathrm{TM}}$ with a kinetics that was initially limited by the surface chemical interaction process. After about 2 hours incubation time (about 130 min.) the kinetics of the adsorption process became limited by the diffusion of reacting species towards the surface thus testifying that the anti-TNF $\alpha$ Affimer ${ }^{\mathrm{TM}}$ almost 


\section{WILEY-VCH}

saturated reactive sites available on the gold surface. The diffusion-limited adsorption process is testified by the observed linear binding rate that replaces the exponential rate observed for analyte-receptor interactions. The diffusion-limited adsorption occurs when the binding rate of an analyte (TNF $\alpha$ in our case) to the receptor (anti-TNF $\alpha$ Affimer $^{\text {TM }}$ in our case) is faster than its diffusion rate to the surface. ${ }^{[62]}$

The reversibility of the recognition process between the immobilized Affimer ${ }^{\mathrm{TM}}$ and TNF $\alpha$ molecule was tested with a control experiment: transfer characteristics for the aptasensor were registered in PBS only and after incubation in $[\mathrm{TNF} \alpha] 500 \mathrm{pM}$, resulting in the previously described decrease in $I_{D S}$. After a second incubation in PBS only, the current response was completely restored to its initial value, as shown in Figure S4 (Supporting Information), thus demonstrating the full reversibility of the interaction. The detection of biorecognition events with FET biosensors also allows for the investigation of the thermodynamics of the process. ${ }^{[9,23,63]}$ Different functions could be used to fit the dose curve in Fig. 2b, viz. the Langmuir function:

$S=S_{\max } \cdot \frac{K_{L} \cdot x}{1+K_{L} \cdot x}$

the Hill function:

$S=S_{\max } \cdot \frac{K_{H}^{\alpha} \cdot x^{\alpha}}{1+K_{H}^{\alpha} \cdot x^{\alpha}}$

and our recently proposed function:

$S=S_{\max } \cdot \frac{\left[C_{w}{ }^{\alpha} \cdot\left(1-\exp \left(-(x / \xi)^{\alpha}\right)\right)+K_{a} \cdot x\right]}{1+\left[C_{w}{ }^{\alpha} \cdot\left(1-\exp \left(-(x / \xi)^{\alpha}\right)\right)+K_{a} \cdot x\right]}$

In both models equation 1 and $\mathbf{2}$, the affinity constants $K_{L}$ and $K_{H}$ represent the inverse TNF $\alpha$ concentration at which $S=S_{\max } / 2 \cdot{ }^{[64,65]}$ In the Hill model (equation 2), the real positive valued exponent $\alpha$ indicates positive $(\alpha>1)$ or negative $(\alpha<1)$ cooperativity that stems from multiple coexisting equilibria. In the model equation 3, the first term embodies the spatial correlation of recognition events, while the second represents a Langmuir-type uncorrelated event. The 


\section{WILEY-VCH}

two terms are additive and their relative weight is measured by $C_{w}{ }^{\alpha} \cdot K_{a}$. In this respect, the affinity constant $K_{a}$ in equation 3 is directly comparable to $K_{L}$ in equation 1 , while the comparison with the Hill model (i.e. an equilibrium constant comparable with $K_{H}$, resulting from the application of equation 2 can be achieved through the following equation:

$K_{H} \cong \frac{C_{w}}{\xi}$

In equation $3, \xi$ is the concentration that marks the crossover between the two regimes, i.e. spatially-correlated and Langmuir-type, uncorrelated events.

We choose the functional described in equation 3 to describe the thermodynamics of the Affimer ${ }^{\mathrm{TM}}$-analyte recognition at the gate, since this functional allows us to take into account, through the $C_{w}$ parameter, the relationship between the recognition events and the electronic structure of both the gate electrode and the organic semiconductor ${ }^{[23]}$. As a comparison, the fitting of the dose curve in Figure 2b with the Langmuir model is provided as Figure $\mathbf{S 8}$ in the Supporting Information.

Using equation 4, we obtain $K_{H}=1.010^{10} \mathrm{M}^{-1}$. This value is comparable with previously reported equilibrium affinity constant for the binding of gold-immobilized Affimer ${ }^{\mathrm{TM}}$ binders with their corresponding cognate antibody. ${ }^{[33,66]}$ On the other hand, this value is one order of magnitude larger than the Hill-like binding constant $\left(1.7 \times 10^{9} \mathrm{M}^{-1}\right)$ that we extract, using the fitting functional described in equation 3, from previous experiments with EGOFET biosensors endowed with monoclonal antibodies against $\mathrm{TNF} \alpha{ }^{[23]}$ The affinity constants displayed by Affimer ${ }^{\mathrm{TM}}$ binders are known to be comparable with those of antibodies. ${ }^{[67,68]}$

The higher $K_{a}$ value for the Affimer ${ }^{\mathrm{TM}} / \mathrm{TNF} \alpha$ pair with respect to the corresponding Antibody/TNF $\alpha$ pair hints to a higher affinity of this specific Affimer compared to the monoclonal $\mathrm{Ab}$ used in our previous experiments, as well as to the reported higher stability of Affimers once immobilized on a substrate. Other factors might also play a role in determining the high sensitivity of these EGOFET peptide aptasensors: for example, the smaller 


\section{WILEY-VCH}

dimensions of the Affimer ${ }^{\mathrm{TM}}$ and the resulting larger coverage on the gate electrode could increase the areal density of analyte binding sites. Please note that because $K_{H}$ is the affinity constant of the binding process at the gate electrode-solution interface, the value found might differ, even by orders of magnitude, from the binding constant measured in solution, since it includes the surface work associated with a molecule binding to a layer of (orderly) immobilized receptors, ${ }^{[9,69]}$ with steric and electrostatic inter-protein interactions altering the overall energetics of the process.

Again, SPRI investigations were performed to validate our approach, with the aim of comparing $K_{a}$ values for the Affimer/TNF $\alpha$ and of Antibody/TNF $\alpha$ pairs. To this aim, we investigated the binding of TNF $\alpha$ with the surface immobilized Affimer ${ }^{\mathrm{TM}}$ by sequentially adsorbing increasingly concentrated TNF $\alpha$ solutions on the Affimer ${ }^{\mathrm{TM}}$ modified SPRI surface. Figure S5 (Supporting Information) shows the average SPRI signal intensity detected after the adsorption of each solution. We used a Langmuir isotherm to fit experimental data shown in Figure S5 by obtaining a binding constant value $\left(K_{a f f}=1.34 \times 10^{11} \mathrm{M}^{-1}\right)$. Similarly, we could obtain via SPRI (Figure S6, Supporting Information) a binding constant value of $K_{a b}=$ $2.47 \times 10^{10} \mathrm{M}^{-1}$ for the binding of TNF $\alpha$ to its corresponding surface immobilized Antibody, following the immobilization procedure described in ref. [23]. The two affinity constant values that we obtain via SPRI differ by about one order of magnitude, in very good agreement with those that we obtain with the EGOFET biosensors, indicating that our platform can indeed serve as a tool for comparing the binding thermodynamics of intermolecular interactions.

Control experiments were performed to assess the specificity of the sensor response. We monitored the interaction between the sensor and a non-specific analyte, the pro-inflammatory cytokine Interleukin-6 (IL-6): exposure of a gate, functionalized with TNF $\alpha$ Affimer ${ }^{\mathrm{TM}}$, to a solution containing $800 \mathrm{pM}$ IL-6, yielded a signal $S=0.05 \pm 0.03$, negligible with respect to 


\section{WILEY-VCH}

the value of $S 0.61 \pm 0.05$ obtained for TNF $\alpha$ at the same concentration (see inset of Figure 2b). We also checked for the response in the absence of gate functionalization, by incubating a bare gold gate to an $800 \mathrm{pM}$ TNF $\alpha$ solution. Again, the signal $\mathrm{S}=0.042 \pm 0.018$ was found to be negligible with respect to the response to that recorded for TNF $\alpha$ at the same concentration with a specific sensing surface, i.e. functionalized with the TNF $\alpha$ Affimer (see inset of Figure $2 b$ ). The response of the EGOFET with non-functionalized gate in the $10 \mathrm{pM}$ $10 \mathrm{nM}$ range is shown in Figure S7, Supporting Information.
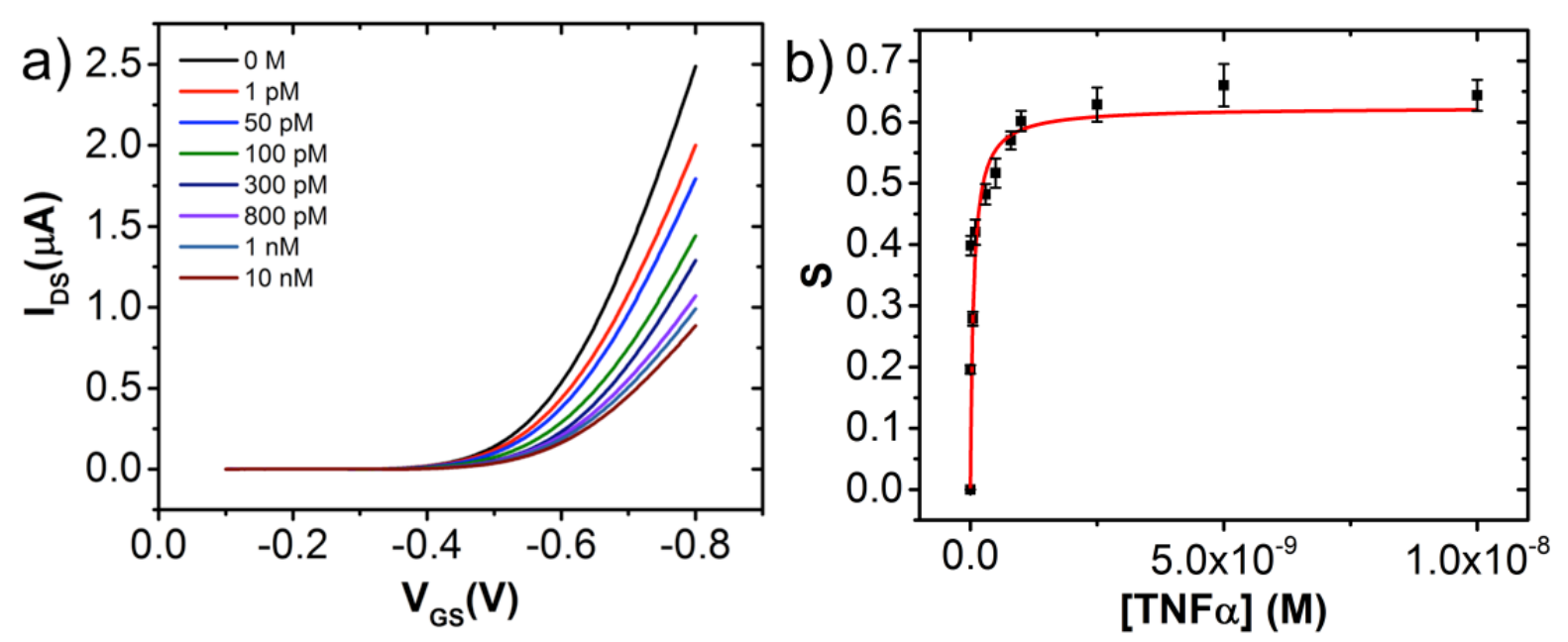

Figure 4. (a) Transfer characteristics of the EGOFET-based sensor resulting from exposure to different $[\mathrm{TNF} \alpha]$ solutions in cell culture medium. The TNF $\alpha$ concentrations are shown in the legend. (b) The corresponding dose curve $S$ vs $[\mathrm{TNF} \alpha]$ acquired at $V_{G S}=-0.8 \mathrm{~V}$. The red line is the best fit for Langmuir functional.

To further assess the selectivity of our EGOFET peptide aptasensor and its operability in real biological samples, we constructed the whole dose curve in a complex matrix such as a cell culture medium. As model, we used the cell culture medium RPMI-1640 enriched with 10\% Fetal Bovine Serum (FBS), antibiotics, amino acids and other small molecules needed for cell 


\section{WILEY-VCH}

proliferation. Cell culture medium and the added FBS are rich of non-specific binders and interfering agents (see Supporting Information for the full composition of the medium), and selective detection in such complex environment well demonstrates the robustness of the sensing architecture. The transfer characteristics $\left(I_{D S}\right.$ vs $V_{G S}$ at a fixed $\left.V_{D S}=-0.2 \mathrm{~V}\right)$ recorded in cell culture medium are shown in Figure 4a, and the corresponding dose curve, extrapolated by calculating the signal $S$ at $V_{D S}=-0.8 \mathrm{~V}$, is shown in Figure $4 \mathrm{~b}$. As expected, the dramatic difference in electrolyte composition (if compared with PBS) is mirrored by differences in the transistor performances, and, in particular, a $V_{t h}$ shift towards more negative values and a decrease of the maximum obtained $I_{D S}$ can be observed; this behavior is not surprising, since the presence in solution of high-molecular-weight, charged moieties influences the structure and the build-up dynamics of the electrical double layer, both at the gate/electrolyte and at the electrolyte/semiconductor interfaces, resulting in a decreased areal charge density under the same biasing conditions. Nonetheless, the evolution of the performances in response to $\mathrm{TNF} \alpha$ in cell culture medium strongly resembles what already observed for PBS, with $I_{D S}$ decreasing with increasing TNF $\alpha$ concentration. Also in this case, the dose curve displays a super-exponential signal increase at $[\mathrm{TNF} \alpha]<300 \mathrm{pM}$ and then reaches a plateau, with a LOD value of $1 \mathrm{pM}$, remarkable for a "real medium". This result is a significant estimator of how our approach could be effectively translated to real human samples.

\section{Conclusions}

In this work, we have demonstrated the use of an EGOFET-based sensor for the detection of the pro-inflammatory cytokine TNF $\alpha$ both in PBS test solutions and in undiluted cell culture media containing $10 \%$ serum. A remarkably low limit of detection could be obtained by using peptide aptamers instead of monoclonal antibodies as the recognition unit. The functionalization strategy is simple, robust and reproducible. The sensor has been operated in 


\section{WILEY-VCH}

a pathological to physiological range of $\mathrm{TNF} \alpha$ concentrations, demonstrating the potential of EGOFET peptide aptasensors in clinical practice. Our strategy can be readily applied to any aptamer/analyte pair, thus opening the way to label-free sub-pM detection (corresponding to $\mathrm{ng} / \mathrm{ml}$ or even lower depending on the target molecular weight) amounts of a large range of targets.

\section{Experimental Section}

\section{Device Fabrication}

The Test Patterns (TPs) $\left(1 \mathrm{~cm}^{2}\right.$ total area) were purchased from "Fondazione Bruno Kessler" (FBK, Trento, Italy). They present 4 interdigitated electrodes with $\mathrm{W} / \mathrm{L}=2000$ (channel length $\mathrm{L}=15 \mu \mathrm{m}$, channel width $\mathrm{W}=30 \mathrm{~mm}$ ) patterned by photolithography and lift-off. The $\mathrm{Au}$ electrodes are $50 \mathrm{~nm}$ thick with a few $\mathrm{nm}$ of $\mathrm{Cr}$ adhesive layer on a quartz substrate, with a roughness lower than $2 \mathrm{~nm}$. The standard cleaning procedure is: (i) a first rinse with acetone (10 ml) in order to remove the photoresist layer, (ii) drying with nitrogen flow, (iii) washing again in hot acetone for $15 \mathrm{~min}$, and (iv) drying with nitrogen. A final rinse with water was done before the semiconductor deposition. Pentacene was deposited by thermal sublimation in high vacuum on TPs held at room temperature (base pressure $10^{-8} \mathrm{mbar}$, rate $5 \AA / \mathrm{min}$ ). The pentacene film was $15 \mathrm{~nm}$ (10 monolayers) for all samples. ${ }^{[23]}$ The polycrystalline Au wire gate electrode cleaning procedure is: (i) immersion in hot $\mathrm{KOH}$ for $4 \mathrm{~h}$, (ii) rinse with abundant water and (iii) immersion in concentrated $\mathrm{H}_{2} \mathrm{SO}_{4}$ at $150{ }^{\circ} \mathrm{C}$ for $2 \mathrm{~h}$.

\section{Gate functionalization}

The gate electrode functionalization procedure is: (i) incubation in anti-TNF $\alpha$ Affimer solution $(0.25 \mathrm{mg} / \mathrm{ml})$ for $12 \mathrm{~h}$ at room temperature, (ii) rinse with abundant water and (iii) incubation in $100 \mu \mathrm{g} / \mathrm{ml}$ BSA solution for $1 \mathrm{~h}$ at room temperature.

\section{Reagents}




\section{WILEY-VCH}

Phosphate salts, sulfuric acid, acetone, ethanol and pentacene were purchased from SigmaAldrich. Recombinant human TNF $\alpha$ was produced by AdipoGen (Liestal, Switzerland) and purchased from Vinci-Biochem S.r.l. (Firenze, Italy). His-tagged anti-TNF $\alpha$ Affimer protein was purchased from Avacta Life Sciences, Ltd (Wetherby, UK).

\section{Electrical Characterization}

Electrical measurements were acquired in buffered solution (PBS $50 \mathrm{mM}, \mathrm{pH}$ 7.4) containing increasing TNF $\alpha$ concentration, ranging from $1 \mathrm{pM}$ to $10 \mathrm{nM}$, under static conditions (i.e. not in a flow cell geometry). The solution was confined in a PDMS pool, as shown in Figure 1a and in Figure S1a, Supplementary Information. Source, drain, and gate electrodes were connected to an Agilent B2902A Source Meter Unit. All measurements were carried out at room temperature inside a Faraday cage (see Figure S1). The $I-V$ transfer characteristics were performed by sweeping the gate-source voltage $\left(V_{G S}\right)$ from +0.2 to $-0.5 \mathrm{~V}$ while leaving the drain-source voltage $\left(V_{D S}\right)$ constant at $-0.2 \mathrm{~V}$ (linear regime). Measurements in complex solutions (i.e., supernatant from monocyte cell cultures) have been performed as described above, in a droplet of the supernatant confined by a PDMS pool. The $I-V$ transfer characteristics were performed by sweeping the gate-source voltage ( $\left.V_{G S}\right)$ from -0.1 to $-0.8 \mathrm{~V}$ while leaving the drain-source voltage $\left(V_{D S}\right)$ constant at $-0.2 \mathrm{~V}$ (linear regime).

We calculated the Limit of Detection (LOD) by obtaining the response of independently measured blank samples (PBS only, with $[\mathrm{TNF} \alpha]=0$, or cell culture medium only for the dose curve in complex biological solutions), and by calculating the corresponding mean and standard deviation $(\sigma \mathrm{d})$ values. We then calculated the LOD as the concentration corresponding to a response that is mean $\pm 3 \sigma \mathrm{d}^{[16]}$, which in our case corresponds to a signal $\mathrm{S}=0.15$ for PBS-based solution and 0.22 for cell culture medium.

Electrochemical characterization 


\section{WILEY-VCH}

A potentiostat/galvanostat PAR model 273A was used for the electrochemical characterization: cyclic voltammetry (CV) experiments were carried out using a threeelectrode cell in argon atmosphere. The working electrode (WE) was a $1 \mathrm{~mm}$ diameter polycrystalline gold wire, the counter electrode (CE) a Pt sheet and the reference electrode (RE) a saturated calomel electrode (SCE). Potentials were calibrated against the $\mathrm{MV}^{2+} / \mathrm{MV}^{+}$ couple (MV methylviologen). The gold WE was cleaned as described above in the text. CV experiments were performed in order to estimate the electrode active area and the coverage after aptamer immobilization by means of the Randles-Sevçik equation ${ }^{[70,71]}$. The measurements have been performed in $2 \mathrm{M} \mathrm{KCl}$ and $5 \mathrm{mM}\left[\mathrm{Fe}(\mathrm{CN})_{6}\right]^{3-/ 4-}$ at $0.020 \mathrm{~V} \mathrm{~s}^{-1}$. $A F M$

Morphological characterization was performed using an NT-MDT SMENA Solver platform (Moscow, Russia); all images were obtained in air in semi-contact mode and analyzed using Gwyddion 2.48 freeware (http://gwyddion.net/). Please note that the electrode used for AFM characterization is different from the one in electrical measurements: to optimize the morphological analysis we used a flat gold electrode which fabrication is described elsewhere. ${ }^{[23]}$

\section{Surface plasmon resonance}

Gold chips for surface plasmon resonance imaging (SPRI) were purchased from Xantec Bioanalytics. We cleaned the chips by leaving them under UV/ozone (5 min.). We washed the cleaned chips with ethanol (10 min.) and dried them under a nitrogen stream.

We used an SPR imager apparatus (GWC Technologies, USA) equipped with a white light source and an SF-10 prism and coupled with a poly(dimethylsiloxane) microfluidic device as elsewhere described [71]. SPR images were obtained by sequentially acquiring 15 frames averaged images with a $15 \mathrm{~s}$ delay between them. A delay of $150 \mathrm{~s}$ was instead introduced to investigate for 12 hours the Affimer ${ }^{\mathrm{TM}}$ immobilization. 


\section{WILEY-VCH}

We obtained sensorgrams by plotting the average pixel values (0-255 gray level scale) referring to the selected regions of interest (ROIs) of the SPR images as a function of time.

SPRI experiments have been performed at room temperature and by filling the microfluidic channel in contact with the SPRI gold surface with the relevant solution. Then the SPRI signals have been acquired under static fluidic conditions. Interactions of TNF $\alpha$ solutions with Affimer ${ }^{\mathrm{TM}}$ or antibody $(\mathrm{Ab})$ were instead performed under continuous flow conditions (flow rate $5 \mu \mathrm{L} \min ^{-1}$ ).

To immobilize Affimer ${ }^{\mathrm{TM}}$ on the surface of the SPRI gold chip, we washed the cleaned chip with PBS (100 mM, pH 6.5) and incubated with anti-TNF $\alpha$ Affimer $^{\mathrm{TM}}(0.25 \mathrm{mg} / \mathrm{ml}$ in PBS $100 \mathrm{mM}, 75 \mathrm{mM} \mathrm{NaCl}, 0.02 \%$ sodium azide- at $\mathrm{pH} 6.5$ ) for 12 h. After the Affimer ${ }^{\mathrm{TM}}$ immobilization and equilibration with PBS buffer, the modified surface was passivated with BSA $(100 \mu \mathrm{g} / \mathrm{ml}$ in PBS $50 \mathrm{mM}$, pH 7.4) for 30 minutes at room temperature.

SPRI chip functionalization with protein $\mathrm{G} / \mathrm{Ab}$ was performed by incubating the cleaned gold surface in His-Tag Protein G solution $(5 \mathrm{mg} / \mathrm{ml}$ in PBS buffer. 30 minutes. Room temperature). The functionalized surface was then washed with PBS and incubated in antiTNF $\alpha$ antibody $(0.1 \mathrm{mg} / \mathrm{ml}$ in PBS, $\mathrm{pH} 7.2,30 \%$ glicerol, $0.5 \%$ BSA, $0.01 \%$ thimerosal $)$ for 1h. The final surface has been rinsed with PBS (50 mM pH 7.4).

We obtained the TNF $\alpha$ solutions ( $1 \mathrm{pM}, 10 \mathrm{pM}, 50 \mathrm{pM}, 100 \mathrm{pM}, 500 \mathrm{pM}, 10 \mathrm{nM})$ used to assess the interaction with Affimer ${ }^{\mathrm{TM}}$ or antibody $(\mathrm{Ab})$ by dilution from a mother solution in PBS. We let TNF $\alpha$ solutions to adsorb on the modified SPRI chip surface by sequentially injecting $\left(5 \mu \mathrm{L} \mathrm{min}^{-1}\right)$ the differently concentrated solutions into the PDMS microfluidic device. We monitored the adsorption of each solution for $16 \mathrm{~min}$.

\section{Acknowledgements}

We gratefully acknowledge IT MIUR Bilateral Project Italy/ Sweden "Poincaré" and the joint 


\section{WILEY-VCH}

INFM-CNR Project "EOS - Organic electronics for innovative measuring instruments" for support. M.P., C.A.B. and F.B. acknowledge support from Life Science Department through "FAR2015". C.A.B. acknowledges the "Fondazione di Vignola" for support. The authors thank Paul Ko Ferrigno (Avacta Life Sciences) for fruitful discussions and help.

Received: ((will be filled in by the editorial staff))

Revised: ((will be filled in by the editorial staff)) Published online: ((will be filled in by the editorial staff))

[1] X. Luo, J. J. Davis, Chem. Soc. Rev. 2013, 42, 5944.

[2] M. L. Hammock, A. N. Sokolov, R. M. Stoltenberg, B. D. Naab, Z. Bao, ACS Nano 2012, 6, 3100 .

[3] H. U. Khan, M. E. Roberts, O. Johnson, W. Knoll, Z. Bao, Org. Electron. physics, Mater. Appl. 2012, 13, 519.

[4] D. Khodagholy, V. F. Curto, K. J. Fraser, M. Gurfinkel, R. Byrne, D. Diamond, G. G. Malliaras, F. Benito-Lopez, R. M. Owens, J. Mater. Chem. 2012, 22, 4440.

[5] M. Magliulo, A. Mallardi, R. Gristina, F. Ridi, L. Sabbatini, N. Cioffi, G. Palazzo, L. Torsi, Anal. Chem. 2013, 85, 3849.

[6] G. Palazzo, D. De Tullio, M. Magliulo, A. Mallardi, F. Intranuovo, M. Y. Mulla, P. Favia, I. Vikholm-Lundin, L. Torsi, Adv. Mater. 2015, 27, 911.

[7] L. Kergoat, B. Piro, M. Berggren, G. Horowitz, M.-C. Pham, Anal. Bioanal. Chem. 2012, 402, 1813.

[8] S. Casalini, F. Leonardi, T. Cramer, F. Biscarini, Org. Electron. 2013, 14, 156.

[9] M. Y. Mulla, E. Tuccori, M. Magliulo, G. Lattanzi, G. Palazzo, K. Persaud, L. Torsi, Nat. Commun. 2015, 6, 6010.

[10] M. Berggren, A. Richter-Dahlfors, Adv. Mater. 2007, 19, 3201.

[11] D. Elkington, N. Cooling, W. Belcher, P. Dastoor, X. Zhou, Electronics 2014, 3, 234. 


\section{WILEY-VCH}

[12] M. L. Hammock, O. Knopfmacher, B. D. Naab, J. B.-H. Tok, Z. Bao, ACS Nano 2013, $7,3970$.

[13] J. T. Mabeck, G. G. Malliaras, Anal. Bioanal. Chem. 2005, 384, 343.

[14] T. Minami, T. Minamiki, Y. Hashima, D. Yokoyama, T. Sekine, K. Fukuda, D. Kumaki, S. Tokito, Chem. Commun. 2014, 50, 15613.

[15] T. Someya, A. Dodabalapur, J. Huang, K. C. See, H. E. Katz, Adv. Mater. 2010, 22, 3799.

[16] L. Torsi, M. Magliulo, K. Manoli, G. Palazzo, Chem. Soc. Rev. 2013, 42, 8612.

[17] L. Torsi, G. M. Farinola, F. Marinelli, M. C. Tanese, O. H. Omar, L. Valli, F. Babudri, F. Palmisano, P. G. Zambonin, F. Naso, Nat. Mater. 2008, 7, 412.

[18] S. Casalini, A. C. Dumitru, F. Leonardi, C. A. Bortolotti, E. T. Herruzo, A. Campana, R. F. de Oliveira, T. Cramer, R. Garcia, F. Biscarini, ACS Nano 2015, 9, 5051.

[19] S. Cotrone, M. Ambrico, H. Toss, M. D. Angione, M. Magliulo, A. Mallardi, M. Berggren, G. Palazzo, G. Horowitz, T. Ligonzo, L. Torsi, Org. Electron. physics, Mater. Appl. 2012, 13, 638 .

[20] L. Kergoat, B. Piro, M. Berggren, M.-C. Pham, A. Yassar, G. Horowitz, Org. Electron. 2012, 13,1 .

[21] S. Lai, M. Barbaro, A. Bonfiglio, Sensors Actuators B Chem. 2016, 233, 314.

[22] M. Magliulo, A. Mallardi, M. Y. Mulla, S. Cotrone, B. R. Pistillo, P. Favia, I. Vikholm-Lundin, G. Palazzo, L. Torsi, Adv. Mater. 2013, 25, 2090.

[23] M. Berto, S. Casalini, M. Di Lauro, S. L. Marasso, M. Cocuzza, D. Perrone, M. Pinti, A. Cossarizza, C. F. Pirri, D. T. Simon, M. Berggren, F. Zerbetto, C. A. Bortolotti, F. Biscarini, Anal. Chem. 2016, 88, 12330.

[24] T. Cramer, a. Campana, F. Leonardi, S. Casalini, a. Kyndiah, M. Murgia, F. Biscarini, J. Mater. Chem. B 2013, 1, 3728.

[25] J. Israelachvili, Intermolecular and Surface Forces, 2011. 


\section{WILEY-VCH}

[26] E. Stern, R. Wagner, F. J. Sigworth, R. Breaker, T. M. Fahmy, M. A. Reed, Nano Lett. 2007, 7, 3405 .

[27] S. Banta, K. Dooley, O. Shur, Annu. Rev. Biomed. Eng. 2013, 15, 93.

[28] A. B. Iliuk, L. Hu, W. A. Tao, Anal. Chem. 2011, 83, 4440.

[29] A. D. Keefe, S. Pai, A. Ellington, Nat. Rev. Drug Discov. 2010, 9, 537.

[30] M. Mascini, I. Palchetti, S. Tombelli, Angew. Chemie Int. Ed. 2012, 51, 1316.

[31] K. Škrlec, B. Štrukelj, A. Berlec, Trends Biotechnol. 2015, 33, 408.

[32] H. Sun, Y. Zu, Molecules 2015, 20, 11959.

[33] J. J. Davis, J. Tkac, S. Laurenson, P. K. Ferrigno, Anal. Chem. 2007, 79, 1089.

[34] J. J. Davis, J. Tkac, R. Humphreys, A. T. Buxton, T. A. Lee, P. Ko Ferrigno, Anal. Chem. 2009, 81, 3314.

[35] P. Estrela, D. Paul, Q. Song, L. K. J. Stadler, L. Wang, E. Huq, J. J. Davis, P. K. Ferrigno, P. Migliorato, Anal. Chem. 2010, 82, 3531.

[36] D. Evans, S. Johnson, S. Laurenson, a G. Davies, P. Ko Ferrigno, C. Wälti, J. Biol. 2008, 7,3 .

[37] A. Johnson, Q. Song, P. Ko Ferrigno, P. R. Bueno, J. J. Davis, Anal. Chem. 2012, 84, 6553.

[38] B. Liu, L. Lu, E. Hua, S. Jiang, G. Xie, Microchim. Acta 2012, 178, 163.

[39] S. R. Shin, Y. S. Zhang, D. Kim, A. Manbohi, H. Avci, A. Silvestri, J. Aleman, N. Hu, T. Kilic, W. Keung, M. Righi, P. Assawes, H. A. Alhadrami, R. A. Li, M. R. Dokmeci, A. Khademhosseini, Anal. Chem. 2016, 88, 10019.

[40] E. S. Forzani, X. Li, P. Zhang, N. Tao, R. Zhang, I. Amlani, R. Tsui, L. A. Nagahara, Small 2006, 2, 1283.

[41] A. Dezieck, O. Acton, K. Leong, E. E. Oren, H. Ma, C. Tamerler, M. Sarikaya, A. K. Y. Jen, Appl. Phys. Lett. 2010, 97, 1.

[42] M. Matmor, N. Ashkenasy, J. Am. Chem. Soc. 2012, 134, 20403. 


\section{WILEY-VCH}

[43] D. Khatayevich, T. Page, C. Gresswell, Y. Hayamizu, W. Grady, M. Sarikaya, Small 2014, 10, 1505 .

[44] H. E. Besar, Kalpana, Ardona, Herdeline Ann, Tovar, John D., Katz, ACS Nano 2015, 9,12401 .

[45] Y. Hayamizu, C. R. So, S. Dag, T. S. Page, D. Starkebaum, M. Sarikaya, Sci. Rep. 2016, 6, 33778 .

[46] T. Goda, Y. Miyahara, Biosens. Bioelectron. 2012, 32, 244.

[47] B. B. Aggarwal, S. C. Gupta, J. H. Kim, Blood 2012, 119, 651.

[48] C. Ley, D. Holtmann, K. M. Mangold, J. Schrader, Colloids Surfaces B Biointerfaces 2011, $88,539$.

[49] Z. Yang, Y. P. Zhao, Eng. Anal. Bound. Elem. 2007, 31, 402.

[50] A. Poghossian, M. J. Schöning, Electroanalysis 2014, 26, 1197.

[51] R. Sharma, S. E. Deacon, D. Nowak, S. E. George, M. P. Szymonik, A. A. S. Tang, D.

C. Tomlinson, A. G. Davies, M. J. McPherson, C. Wälti, Biosens. Bioelectron. 2016, 80, 607.

[52] R. Kolodziejczyk, K. Michalska, A. Hernandez-Santoyo, M. Wahlbom, A. Grubb, M. Jaskolski, FEBS J. 2010, 277, 1726.

[53] T. Minami, T. Sato, T. Minamiki, K. Fukuda, D. Kumaki, S. Tokito, Biosens. Bioelectron. 2015, 74, 45.

[54] M. Magliulo, D. De Tullio, I. Vikholm-Lundin, W. M. Albers, T. Munter, K. Manoli, G. Palazzo, L. Torsi, Anal. Bioanal. Chem. 2016, 408, 3943.

[55] M. Di Lauro, S. Casalini, M. Berto, A. Campana, T. Cramer, M. Murgia, M. Geoghegan, C. A. Bortolotti, F. Biscarini, ACS Appl. Mater. Interfaces 2016, 8, 31783.

[56] O. Arican, M. Aral, S. Sasmaz, P. Ciragil, Mediators Inflamm. 2005, 2005, 273.

[57] S. K. Arya, P. Estrela, Methods 2016, 1.

[58] A. Ferrajoli, M. J. Keating, T. Manshouri, F. J. Giles, A. Dey, Z. Estrov, C. A. Koller, 


\section{WILEY-VCH}

R. Kurzrock, D. A. Thomas, S. Faderl, S. Lerner, S. O. Brien, M. Albitar, Blood 2012, $100,1215$.

[59] L. Gibellini, S. De Biasi, E. Bianchini, R. Bartolomeo, A. Fabiano, M. Manfredini, F. Ferrari, G. Albertini, T. Trenti, M. Nasi, M. Pinti, A. Iannone, C. Salvarani, A.

Cossarizza, G. Pellacani, PLoS One 2016, 11, e0167757.

[60] Y.-S. Moon, D.-H. Kim, D.-K. Song, Metabolism. 2004, 53, 863.

[61] M. Pinti, E. Cevenini, M. Nasi, S. De Biasi, S. Salvioli, D. Monti, S. Benatti, L. Gibellini, R. Cotichini, M. A. Stazi, T. Trenti, C. Franceschi, A. Cossarizza, Eur. J. Immunol. 2014, 44, 1552.

[62] R. L. Rich, D. G. Myszka, J. Mol. Recognit. 2008, 21, 355.

[63] M. Larisika, C. Kotlowski, C. Steininger, R. Mastrogiacomo, P. Pelosi, S. Schütz, S. F. Peteu, C. Kleber, C. Reiner-Rozman, C. Nowak, W. Knoll, Angew. Chemie Int. Ed. $\mathbf{2 0 1 5}, 54,13245$.

[64] S. Goutelle, M. Maurin, F. Rougier, X. Barbaut, L. Bourguignon, M. Ducher, P. Maire, Fundam. Clin. Pharmacol. 2008, 22, 633.

[65] J. N. Weiss, FASEB J. 1997, 11, 835.

[66] S. Straw, P. K. Ferrigno, Q. Song, D. Tomlinson, F. Del Galdo, J. Biomed. Sci. Eng. 2013, 6,32 .

[67] L. K. J. Stadler, T. Hoffmann, D. C. Tomlinson, Q. Song, T. Lee, M. Busby, Y. Nyathi, E. Gendra, C. Tiede, K. Flanagan, S. J. Cockell, A. Wipat, C. Harwood, S. D. Wagner, M. A. Knowles, J. J. Davis, N. Keegan, P. Ko Ferrigno, Protein Eng. Des. Sel. 2011, 24,751 .

[68] C. Tiede, A. A. S. Tang, S. E. Deacon, U. Mandal, J. E. Nettleship, R. L. Owen, S. E. George, D. J. Harrison, R. J. Owens, D. C. Tomlinson, M. J. McPherson, Protein Eng. Des. Sel. 2014, 27, 145.

[69] G. Oliviero, S. Federici, P. Colombi, P. Bergese, J. Mol. Recognit. 2011, 24, 182. 


\section{WILEY-VCH}

[70] S. Casalini, F. Leonardi, C. a. Bortolotti, A. Operamolla, H. Omar, L. Paltrinieri, C.

Albonetti, M. Farinola, F. Biscarini, O. H. Omar, L. Paltrinieri, C. Albonetti, G. M.

Farinola, F. Biscarini, J. Mater. Chem. 2012, 22, 12155.

[71] S. Casalini, M. Berto, F. Leonardi, A. Operamolla, C. a Bortolotti, M. Borsari, W. Sun, R. Di Felice, S. Corni, C. Albonetti, O. H. Omar, G. M. Farinola, F. Biscarini, Langmuir 2013, 29, 13198.

An organic electronics device for the label-free detection of inflammatory cytokine $\mathbf{T N F} \boldsymbol{\alpha}$ is presented. The device is based on the EGOFET architecture, and the sensing unit is based on the use of peptide aptamers immobilized on the gold gate electrode. The biosensor is demonstrated in PBS and cell culture medium, exhibiting $1 \mathrm{pM}$ limit of detection and remarkable selectivity.

Organic bioelectronics; peptide aptamers; Affimer protein; TNF $\alpha$; binding constant M. Berto, C. Diacci, R. D’Agata, M. Pinti, E. Bianchini, M. Di Lauro, S. Casalini, A. Cossarizza, M. Berggren, D. Simon, G. Spoto, F. Biscarini*, C. A. Bortolotti*

EGOFET peptide aptasensor for label-free detection of inflammatory cytokines in complex fluids

ToC figure

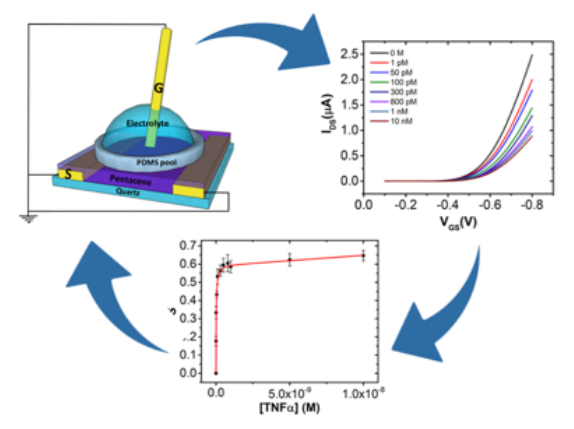

